\title{
Evaluation of the Calculated Sizes Based on the Neural Network Regression
}

\author{
Zexiang Zhao $\mathbb{D}^{\mathrm{D}},{ }^{1}$ Jianpu Xi, ${ }^{1}$ Xinyu Zhao, ${ }^{2}$ Guoqing Zhang, ${ }^{1}$ and Mengjiao Shang ${ }^{1}$ \\ ${ }^{1}$ School of Mechatronics Engineering, Zhongyuan University of Technology, Zhengzhou 450007, China \\ ${ }^{2}$ School of Computer Science, Zhongyuan University of Technology, Zhengzhou 450007, China \\ Correspondence should be addressed to Zexiang Zhao; zexiang_zhao@zut.edu.cn
}

Received 10 May 2018; Accepted 28 August 2018; Published 2 October 2018

Academic Editor: Dimitris Mourtzis

Copyright (c) 2018 Zexiang Zhao et al. This is an open access article distributed under the Creative Commons Attribution License, which permits unrestricted use, distribution, and reproduction in any medium, provided the original work is properly cited.

\begin{abstract}
The evaluation models of circumference diameter, area diameter, and volume diameter were established based on the cylindrical coordinate measuring method, respectively. Many groups of roundness profiles of cylindrical specimens were extracted, and their pseudo and actual circumference diameters, area diameters, and volume diameters were evaluated according to the established evaluation models and the sampling data of the extracted roundness profiles. The relationship models between the pseudo calculated sizes and the actual calculated sizes were built through a series of training based on the neural network regression. The checked experiments for the training models showed that the evaluation of the calculated sizes can meet their measurement accuracy requirement through the pseudo calculated sizes, which were evaluated based on the roundness profiles by using the cylindricity or roundness measuring instrument.
\end{abstract}

\section{Introduction}

Besides the global sizes, two-point size, and ball size, the calculated sizes of cylinder are defined in ISO 14405-1, which are circumference diameter, area diameter, and volume diameter [1]. In the standard, there are no measurement methods for the calculated sizes of cylinder. Through analyzing the definition of the calculated sizes of cylinder, the precision cylindrical coordinate measuring machine is an instrument suitable for measurement of these sizes. Owing to the fact that the absolute sizes between the sampling points in the cylinder's profile and the rotary axis of the worktable should be measured for the realization of precision measurement of the calculated sizes, although ISO 14405-1 was issued by ISO (International Standardization Organization), there have been no suitable products of precision cylindrical coordinate measuring machine $\left(\mathrm{C}^{2} \mathrm{M}^{2}\right)$ at present, and the standard can not be implemented in the real production. On the other hand, the roundness error of cylinder can be measured by using precision cylindricity or roundness measuring instruments [2-6], which can be seen as a like cylindrical coordinate measuring machine (Like- $\mathrm{C}^{2} \mathrm{M}^{2}$ ) in this paper. Owing to that the variations of the sampling points in the extracted roundness profile [7-10] by using
Like- $\mathrm{C}^{2} \mathrm{M}^{2}$ are the relative radial sizes, the roundness error or cylindricity error can be evaluated based on the pseudo roundness profile, the pseudo radial sizes of which are equal to the sum values of the radial variations of the sampling points and a radius of a virtual ideal cylinder. Besides the evaluation of the roundness and cylindricity errors [11-13], the pseudo calculated sizes can also be obtained based on the pseudo radial sizes above. The actual calculated sizes can be obtained based on the trained neural network regression of the pseudo calculated sizes and the actual calculated sizes. The trained models for the evaluation of the calculated sizes were checked. The checked results showed that this method can satisfy the measuring accuracy requirement of the calculated sizes, which may be one way to solve the measurement problem of the calculated sizes of cylinder for implementing ISO 14405-1 in the manufacturing industry as soon as possible.

\section{Evaluation Models of the Calculated Sizes of Cylinder}

2.1. Evaluation Model of the Circumference Diameter. The ith pseudo or actual roundness profile of cylinder is extracted by 


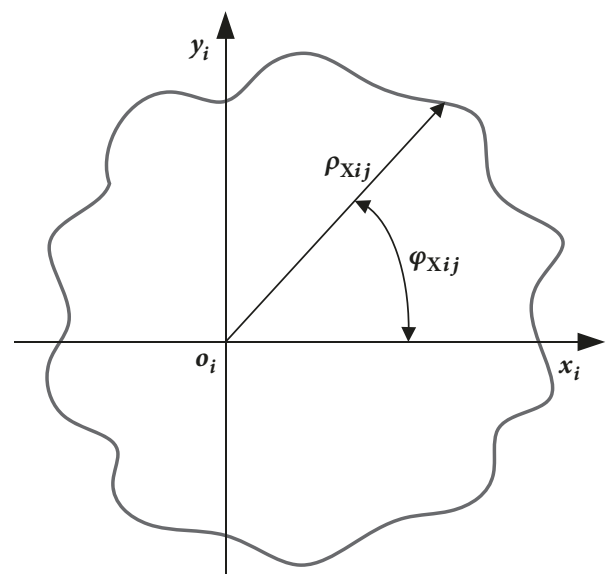

FIGURE 1: The ith pseudo or actual roundness profile of cylinder.

using Like- $\mathrm{C}^{2} \mathrm{M}^{2}$ or $\mathrm{C}^{2} \mathrm{M}^{2}$, as shown in Figure 1, where the subscript "X" of $\rho_{\mathrm{X} i j}$ and $\varphi_{\mathrm{X} i j}$ can be expressed as "p" and "a" for the pseudo and actual roundness profiles, respectively, and $\rho_{\mathrm{p} i j}$ is equal to the sum of the relative variation of the $j$ th sampling point in the $i$ th roundness profile extracted by using Like- $\mathrm{C}^{2} \mathrm{M}^{2}$ and a radius of a virtual cylinder, while $\rho_{\mathrm{aij}}$ is the actual radial size of the $j$ th sampling point in the $i$ th roundness profile extracted by using $\mathrm{C}^{2} \mathrm{M}^{2}$.

According to the definition of the circumference diameter in ISO 14405-1, the circumference diameter $D_{\mathrm{XP} i}$ of the $i$ th roundness profile can be gained as follows:

$$
D_{\mathrm{XP} i}=\frac{P_{\mathrm{X} i}}{2 \pi}
$$

where $P_{\mathrm{X} i}$ is the circumference of the $i$ th pseudo or actual roundness profile, as shown in Figure 2, which can be determined as follows:

$$
P_{\mathrm{X} i}=\sum_{j=1}^{n} \Delta P_{\mathrm{X} i j}
$$

where $\Delta P_{\mathrm{X} i j}, j=1 \sim n-1$, is the distance of the $j$ th sampling point $S_{i j}$ and $(j+1)$ th sampling point $S_{i(j+1)}$ in the $i$ th pseudo or actual roundness profile, where $n$ is the number of the sampling points and $\Delta P_{\text {Xin }}$ is the distance of the $n$th and $1^{\text {st }}$ sampling points in the $i$ th pseudo or actual roundness profile.

The cosine law is used for triangle $\Delta o_{i} S_{\mathrm{X} i j} S_{\mathrm{X} i(j+1)}$, as shown in Figure 3, where $\Delta \varphi_{\mathrm{X} i j}=\varphi_{\mathrm{X} i(j+1)}-\varphi_{\mathrm{X} i j}$, and then $\Delta P_{\mathrm{X} i j}$ can be calculated as follows:

$$
\Delta P_{\mathrm{X} i j}=\sqrt{\rho_{\mathrm{X} i(j+1)}^{2}+\rho_{\mathrm{X} i j}^{2}-2 \rho_{\mathrm{X} i(j+1)} \rho_{\mathrm{X} i j} \cos \Delta \varphi_{\mathrm{X} i j}}
$$

2.2. Evaluation Model of the Area Diameter. According to the definition of the area diameter in ISO 14405-1, the area diameter $D_{\mathrm{XA} i}$ of the $i$ th pseudo or actual roundness profile can be obtained as follows:

$$
D_{\mathrm{XA} i}=2 \sqrt{\frac{A_{\mathrm{X} i}}{\pi}}
$$

where $A_{\mathrm{X} i}$ is the area of the $i$ th pseudo or actual roundness profile, which can be determined as follows:

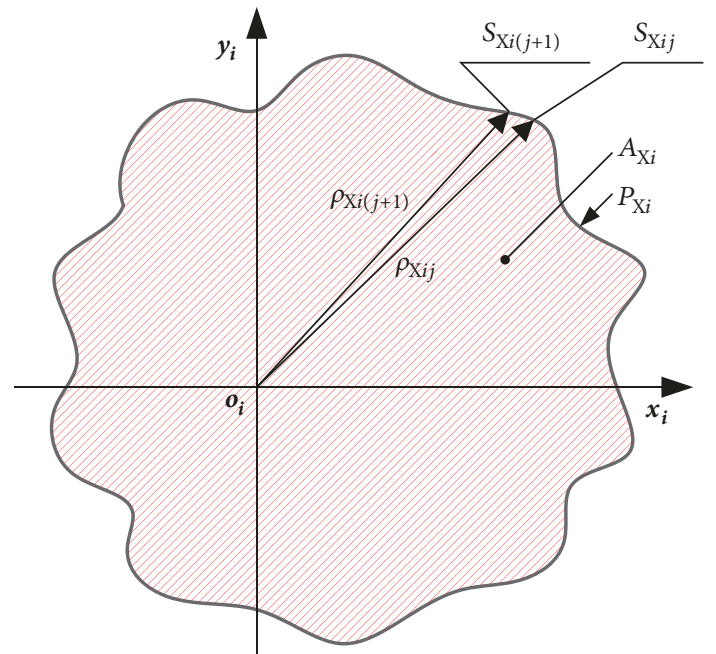

FIgURE 2: Schematic of the circumference $P_{\mathrm{X} i}$ and the area $A_{\mathrm{X} i}$ of the $i$ th roundness profile.

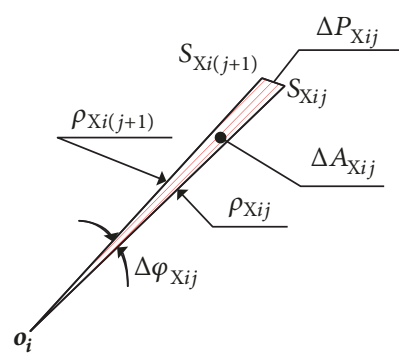

FIGURE 3: Schematic of triangle $\Delta o_{i} S_{\mathrm{X} i j} S_{\mathrm{X} i(j+1)}$.

$$
A_{\mathrm{X} i}=\sum_{j=1}^{n} \Delta A_{\mathrm{X} i j}
$$

where $\Delta A_{\mathrm{X} i j}$ is the area of triangle $\Delta o_{i} S_{\mathrm{X} i j} S_{\mathrm{X} i(j+1)}$ shown in Figure 3, which can be calculated as follows:

$$
\Delta A_{\mathrm{X} i j}=\frac{1}{2} \rho_{\mathrm{X} i j} \rho_{\mathrm{X} i(j+1)} \sin \Delta \varphi_{\mathrm{X} i j}
$$




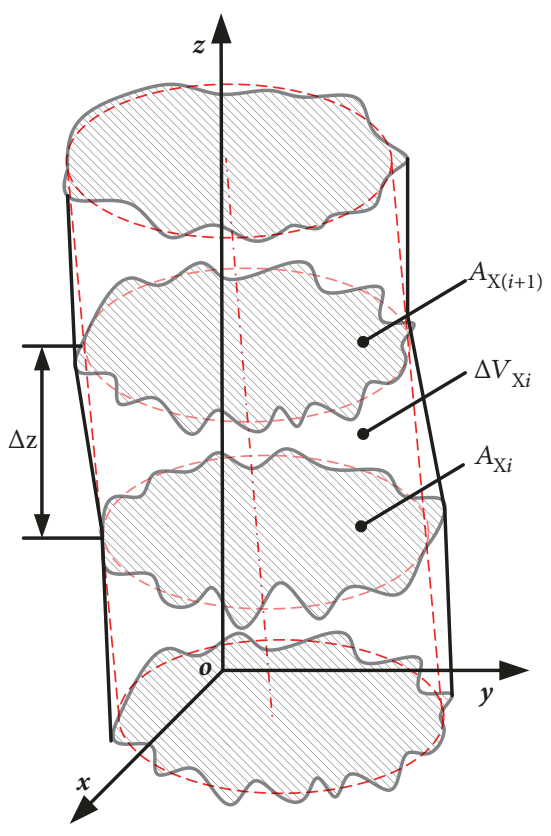

FIgURE 4: Schematic of the calculation $\Delta V_{\mathrm{X} i}$ of cylinder.

2.3. Evaluation Model of the Volume Diameter. According to the definition of the volume diameter in ISO 14405-1, the volume diameter $D_{\mathrm{Xv}}$ of cylinder can be calculated as follows:

$$
D_{\mathrm{XV}}=2 \sqrt{\frac{V_{\mathrm{X}}}{\pi L}}
$$

where $V_{\mathrm{X}}$ is the pseudo and actual volume of cylinder with the measured length $L$, which is the distance of the $n$th roundness profile and the $1^{\text {st }}$ roundness profile along $z$-axis, as shown in Figure 4.

According to (5), $A_{\mathrm{X} i}, i=1 \sim n$, can be calculated for all pseudo or actual roundness profiles, and $V_{\mathrm{X}}$ can be obtained as follows:

$$
V_{\mathrm{X}}=\sum_{i=1}^{m-1} \Delta V_{\mathrm{X} i}
$$

where $\Delta V_{\mathrm{X} i}$ is the pseudo or actual volume between the $i$ th roundness profile and the $(i+1)$ th roundness profile and the distance of both roundness profiles along $z$-axis is $\Delta z$, which is equal to $L /(m-1)$, where $m$ is the number of the roundness profiles of the measured cylinder. $\Delta V_{\mathrm{X} i}$ can be obtained as follows:

$$
\Delta V_{\mathrm{X} i}=\left(A_{\mathrm{X} i}+A_{\mathrm{X}(i+1)}\right) \times \frac{\Delta z}{2}
$$

\section{The Neural Network Regression of the Pseudo Calculated Sizes and the Actual Calculated Sizes}

Although $\rho_{\mathrm{Xij}}$ is used as the basis of evaluation of the form errors and the calculated sizes of cylinder based on the roundness profile extraction strategy, its meaning is different.

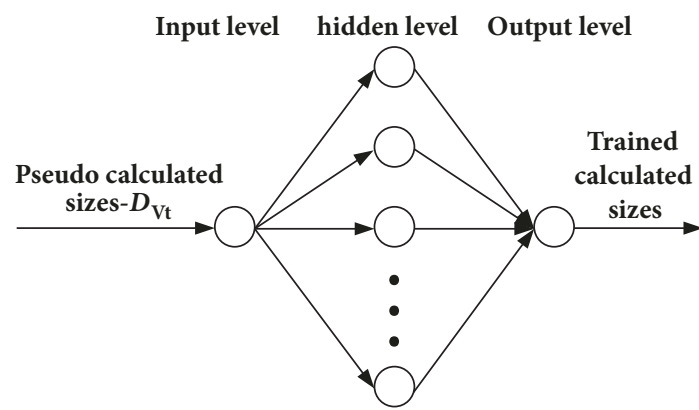

FIGURE 5: Schematic of the neural network regression with three levels.

For the measurement of $\rho_{\mathrm{p} i j}$ in the evaluation of the form errors, it is unnecessary that $\rho_{\text {pij }}$ should be the actual distance from the sampling point to the rotary axis of the worktable, which may be equal to the sum of $\delta_{i j}$ and $D_{\mathrm{vt}} / 2$, which is half of the nominal size of the measured cylinder or a radius of a virtual cylinder, while $\delta_{i j}$ is the relative radial variation of the $j$ th sampling point in the $i$ th roundness profile with high precision, which is usually measured by using Like$\mathrm{C}^{2} \mathrm{M}^{2}$, such as the cylindricity measuring instrument and the roundness measuring instrument. For the measurement of $\rho_{\text {aij }}$ in the evaluation of the calculated sizes, it is necessary that $\rho_{\mathrm{a} i j}$ should be the actual distance from the sampling point to the rotary axis of the worktable, which is usually measured by using the $\mathrm{C}^{2} \mathrm{M}^{2}$.

For the establishment of the relationship between the pseudo calculated sizes and the actual calculated sizes, the nonlinear regression based on the neural network with three levels was used, which include an input level, a hidden level, and an output level, as shown in Figure 5.

For the circumference diameter, the area diameter, and the volume diameter, input level mainly consists of one node, that is, the difference between their respective pseudo diameter and the diameter $D_{\mathrm{vt}}$ of a virtual cylinder, there are many nodes in hidden level, which can be determined by a series of the model training, and the output level has one node, in which the trained diameters in Figure 5 can be gained.

For the neural networks in Matlab, "trainlm", "trainbr", and "trainscg" functions are often selected. The former two functions are based on Levenberg-Marquardt optimization, while the third function is based on the scaled conjugate gradient method. The three training functions' algorithms can be seen in $[14,15]$.

\section{Experiments and Results}

4.1. Experiment Design. For the circumference diameter or the area diameter, 835 roundness profiles of 49 ground cylindrical workpieces with a nominal size of $\phi 40 \mathrm{~mm}$ were extracted by using a global size measuring instrument, developed by Zhongyuan University of Technology. For verifying the feasibility of evaluating the calculated sizes based on the trained models of the neural network regression between the pseudo calculated sizes and the actual calculated sizes, the diameter $D_{\mathrm{vt}}$ of the virtual cylinder was selected as $20 \mathrm{~mm}$, 


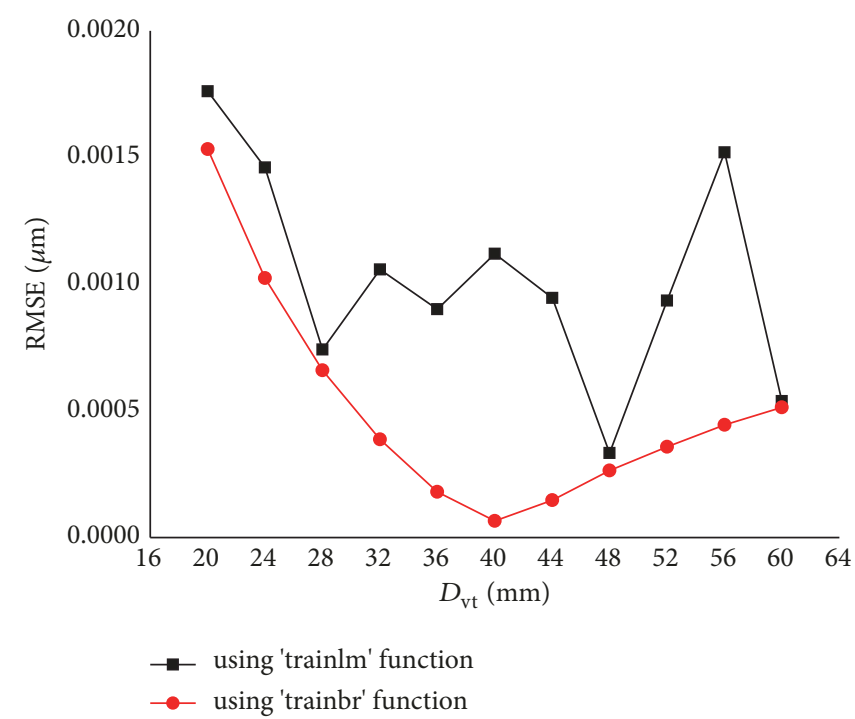

(a) Minimum RMSE between the trained and actual circumference diameters

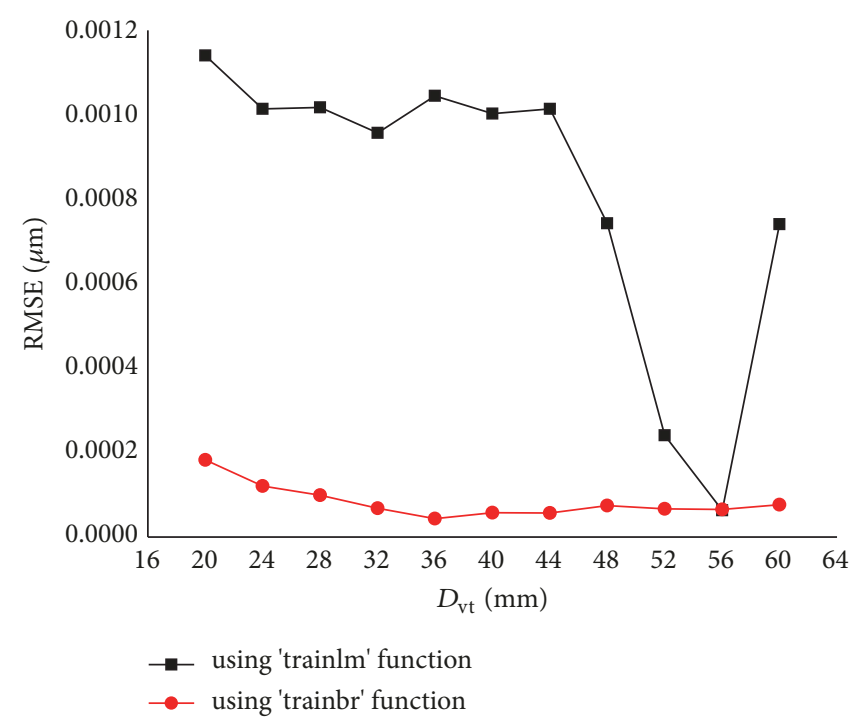

(b) Minimum RMSE between the trained and actual area diameters

FIGURE 6: Minimum RMSE of the circumference diameters and the area diameters.

$24 \mathrm{~mm}, 28 \mathrm{~mm}, 32 \mathrm{~mm}, 36 \mathrm{~mm}, 40 \mathrm{~mm}, 44 \mathrm{~mm}, 48 \mathrm{~mm}, 52 \mathrm{~mm}$, $56 \mathrm{~mm}$, and $60 \mathrm{~mm}$, respectively, the purpose of which is to check out the influence of the different virtual diameters $D_{\mathrm{vt}}$ on the evaluation results of the calculated sizes based on the trained models of the neural network regression.

For the neural network in Figure 5, the training parameters were set as follows: the maximum epochs were 1000 , the train, validation, and test ratios were $0.7,0.15$, and 0.15 , respectively, the maximum number of the fail epochs was 6 , the train goal was $1.0 \mathrm{e}-12$, and the "trainlm" and "trainbr" training functions were used for the evaluation models of the circumference diameter and the area diameter, while three training functions above were used for the evaluation models of the volume cylinder. For the hidden level's nodes from 3 to 15 , the neural network nonlinear regression of circumference diameter, area diameter, and volume diameter were carried out for 100 times, respectively, and the neural network optimal nonlinear regression models of the circumscribed diameter, the area diameter, and the volume diameter were selected based on the MSE (Mean Square Error) values of output values and target values for simplification, respectively.

4.2. Experiment Results. The minimum values of RMSE (Root Mean Square Error) of the output values and their target values of the circumference diameters and the area diameters for the above values of $D_{\mathrm{vt}}$ under two training functions were shown in Figure 6, and the minimum values of RMSE of the output values and their target values of the volume diameters for the above values of $D_{\mathrm{vt}}$ under three training functions were shown in Figure 7. The minimum value of RMSE refers to the minimum value among the 1300 RMSE values obtained under 1300 training times from 3 to 15 hidden level's nodes for each $D_{\mathrm{vt}}$. The hidden nodes obtaining the minimum RMSE values under the diameters $D_{\mathrm{vt}}$ of the virtual cylinders can be seen in Table 1.
From Figures 6 and 7 and Table 1, we can get the following conclusions: All the minimum RMSE values between the outputs of the trained models by using two training functions for the circumference and area diameters and three training functions for the volume diameters are able to meet their measuring accuracy requirement for all the virtual cylinders with the diameters from $20 \mathrm{~mm}$ to $60 \mathrm{~mm}$, but for the calculated sizes, their evaluation results of the models trained by using the "trainbr" function are superior to the other two functions. The nodes of hidden levels of the three training functions for three kinds of calculated sizes have no explicit relationships with the diameters $D_{\mathrm{vt}}$ of the virtual cylinders.

For further checking the feasibility estimating the calculated sizes by using the trained models above, the 260 roundness profiles of 14 cylinders with a nominal size of $25 \mathrm{~mm}$ were adopted. For the corresponding relationship to $D_{\mathrm{vt}}$ in Table 1 , a series of the checked virtual cylinders with diameters $D_{\mathrm{vtc}}$ from $12.5 \mathrm{~mm}$ to $37.5 \mathrm{~mm}$, where the difference between two adjacent checked virtual cylinders is $2.5 \mathrm{~mm}, 260$ pseudo circumference diameters, 260 pseudo area diameters, and 14 pseudo volume diameters for each 260 and their corresponding actual calculated sizes with a nominal sizes of $25 \mathrm{~mm}$ were evaluated based on (1), (4), and (7).

Their corresponding circumference diameters, area diameters, and volume diameters were evaluated based on the trained models used in Figures 7 and 8 for all checked virtual cylinders, and their corresponding RMES values between the outputs (the calculated sizes evaluated by trained models) and the actual calculated sizes (targets) for the checked virtual cylinders were obtained, as shown in Figure 8 for the circumference and area diameters and in Figure 9 for the volume diameters.

From Figure 8(a), we can know that the RMSE values obtained based on the trained models by using "trainlm" and "trainbr" functions and the diameters $D_{\mathrm{vtc}}$ of all the checked virtual cylinders can meet the measuring accuracy 


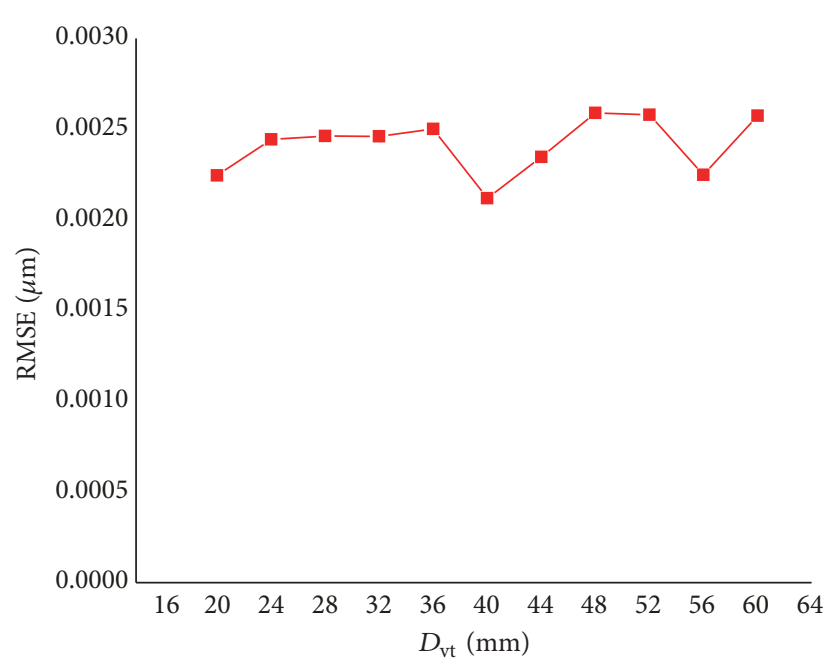

(a) Minimum RMSE of the volume diameter based on the "trainlm" function

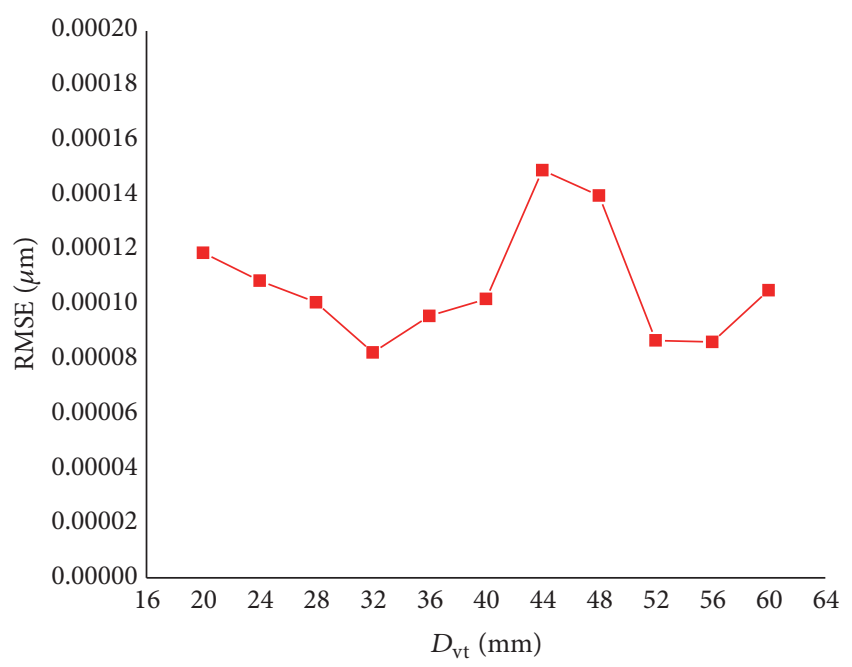

(b) Minimum RMSE of the volume diameter based on the "trainbr" function

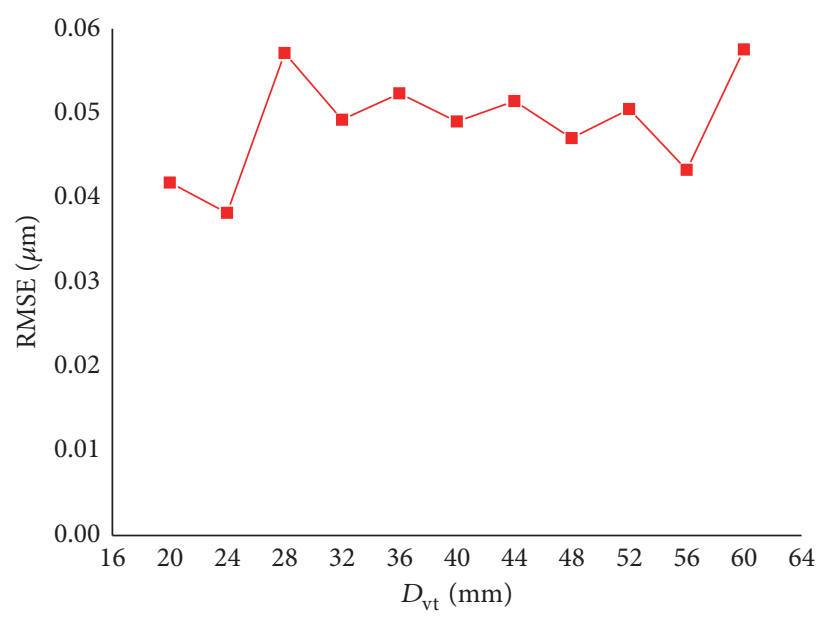

(c) Minimum RMSE of the volume diameter based on the "trainscg" function

FIGURE 7: Minimum RMSE of the volume diameters.

requirements of the circumference diameters, and from Figure 8(b), we can know that the RMSE values obtained based on the trained models by using "trainbr" function and the diameters $D_{\text {vtc }}$ of all the checked virtual cylinders can meet the measuring accuracy requirements of the area diameters, and except for the checked virtual cylinder with a diameter $D_{\text {vtc }}$ of $15 \mathrm{~mm}$, the RMSE values obtained based on the trained models by using "trainlm" function and the diameters $D_{\text {vtc }}$ of all the other checked virtual cylinders can meet the measuring accuracy requirements of the area diameters. From Figure 9, we can know that the RMES values obtained based on the trained models by using "trainbr" functions and the diameters $D_{\mathrm{vtc}}$ of all the checked virtual cylinders can meet the measuring accuracy requirements of the volume diameters.

In conclusion, for three kinds of calculated sizes, their evaluation based on the neural network regression models trained by using "trainbr" function is feasible and has higher evaluation accuracy.
4.3. Results Analysis. For further analyzing the results above, we took the neural network regression curves of the calculated sizes of the virtual cylinder with the diameter $D_{\mathrm{vt}}$ of $36 \mathrm{~mm}$ and the checked virtual cylinder with the diameter $D_{\text {vtc }}$ of $22.5 \mathrm{~mm}$ as an example of evaluating the calculated sizes using the neural network regression models trained by using "tainbr" function. For 835 roundness profiles of 49 cylinders with a nominal size of $40 \mathrm{~mm}$, Figures 10 (a), $10(\mathrm{c})$, and 10 (e) are the regression curves between the outputs and their corresponding targets, where the outputs are the calculated sizes obtained based on the trained models and the pseudo calculated sizes of the virtual cylinder with the diameter $D_{\mathrm{vt}}$ of $36 \mathrm{~mm}$, and the targets are their corresponding actual calculated sizes. Figures 10(b), 10(d), and 10(f) are the distribution histograms of the errors between the outputs and their corresponding targets.

For the 260 roundness of 14 cylinders with a nominal size of $25 \mathrm{~mm}$, Figures 11(a), 11(c), and 11(e) are the regression curves between the outputs and their corresponding targets, 


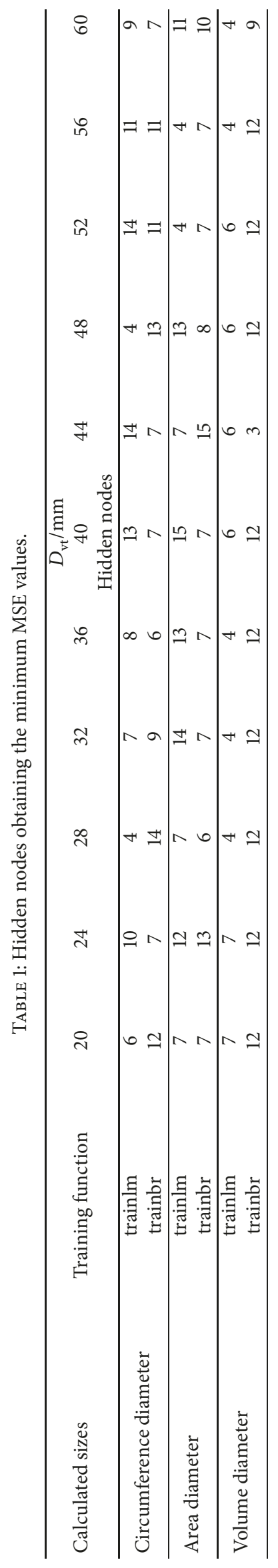




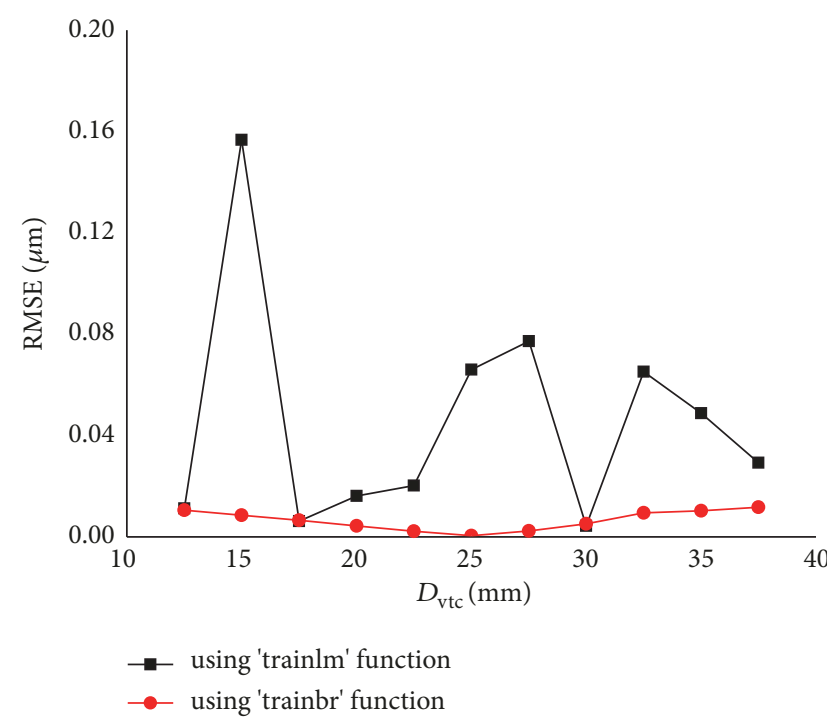

(a) RMSE of the circumference diameters by using the models in Figure 6(a)

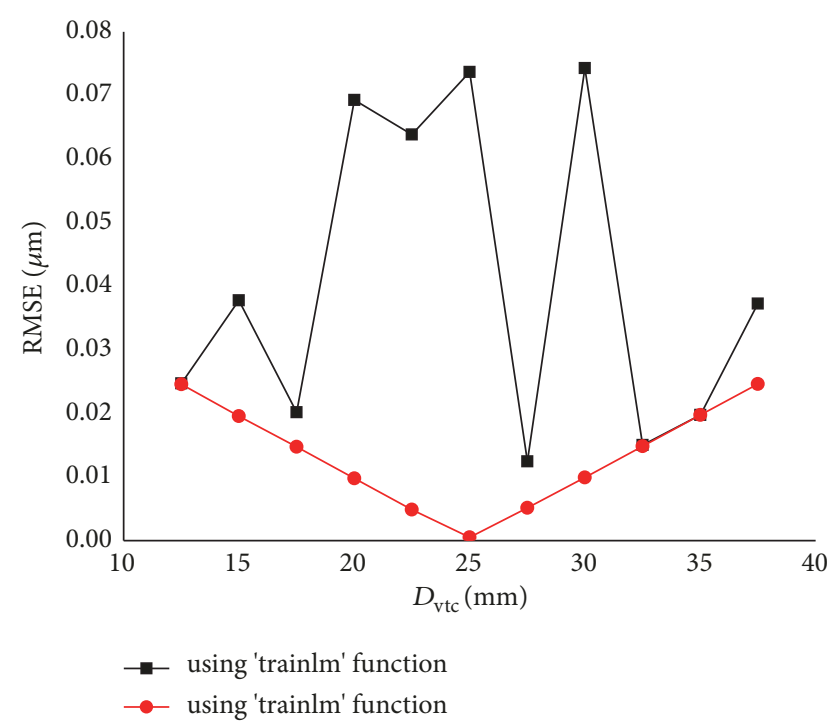

(b) RMSE of the area diameters by using the models in Figure 6(b)

FIGURE 8: RMSE of the circumference diameters and the area diameters of the checked cylinders.

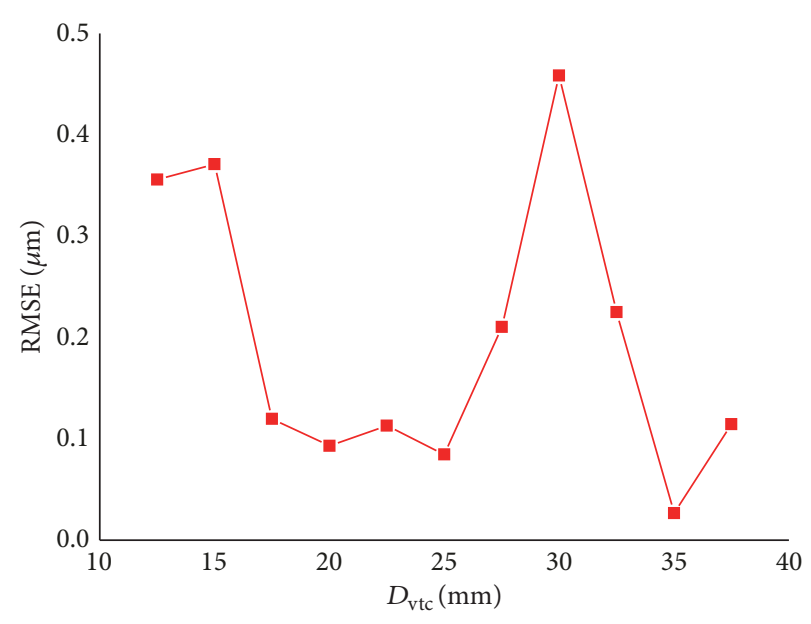

(a) RMSE of the volume diameters evaluated by using the models in Figure 7(a)

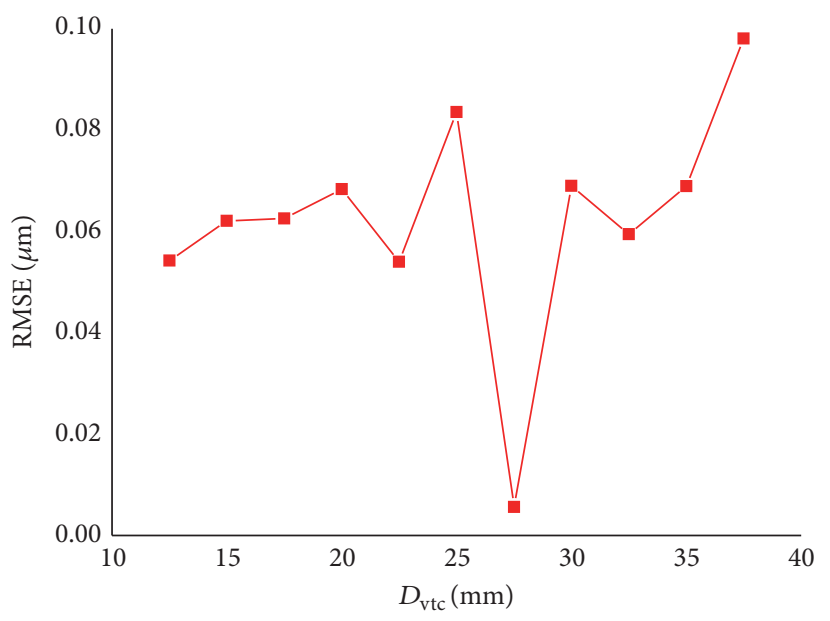

(b) RMSE of the volume diameters evaluated by using the models in Figure 7(b)

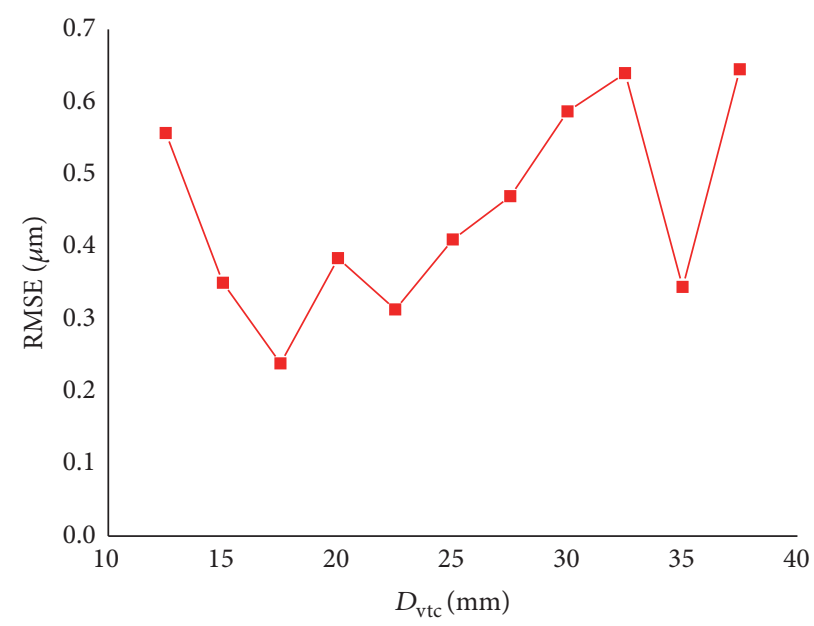

(c) RMSE of the volume diameters evaluated by using the models in Figure 7(c)

FIGURE 9: RMSE of the volume diameters of the checked cylinders. 


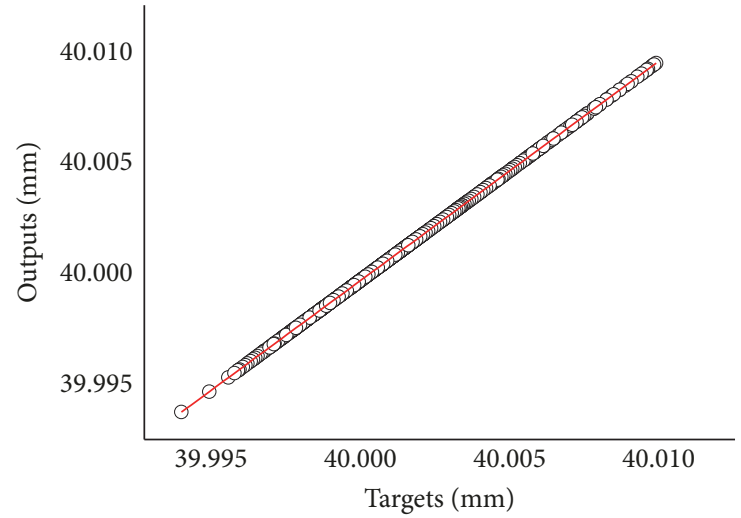

○ Outputs

_- Fit line

(a) Regression curve for $D_{\mathrm{P}}$

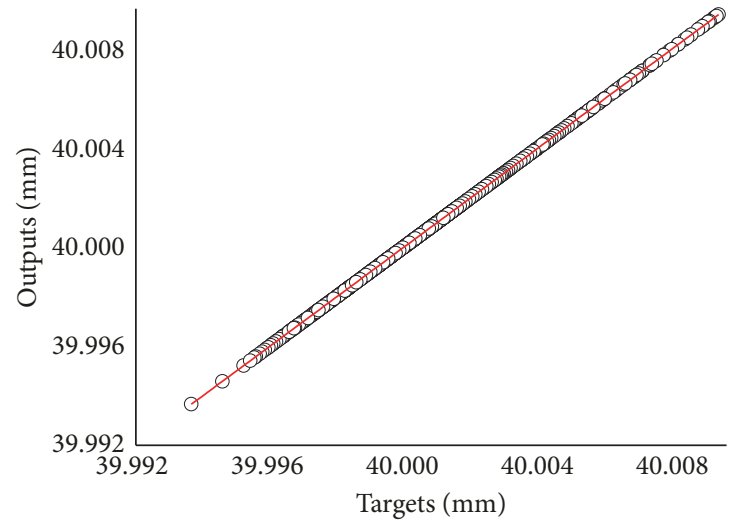

○ Outputs

- Fit line

(c) Regression curve for $D_{\mathrm{A}}$

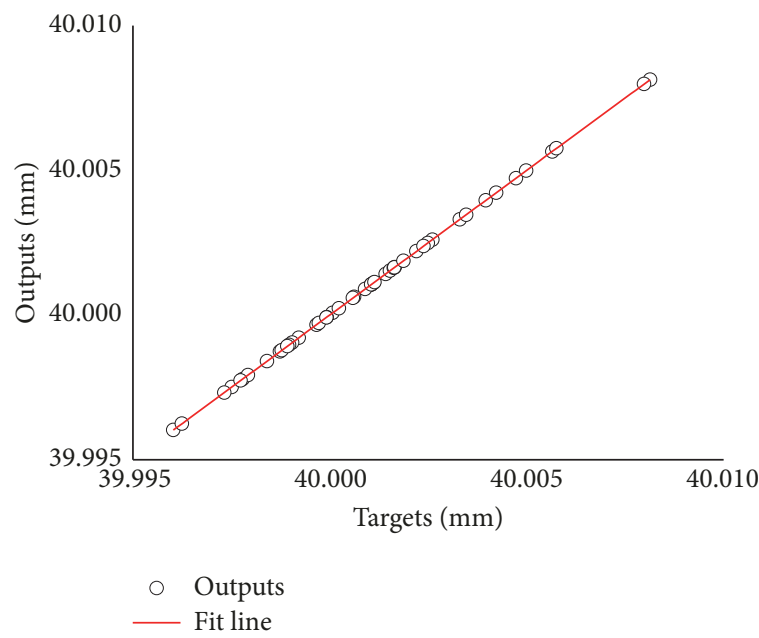

(e) Regression curve for $D_{\mathrm{V}}$

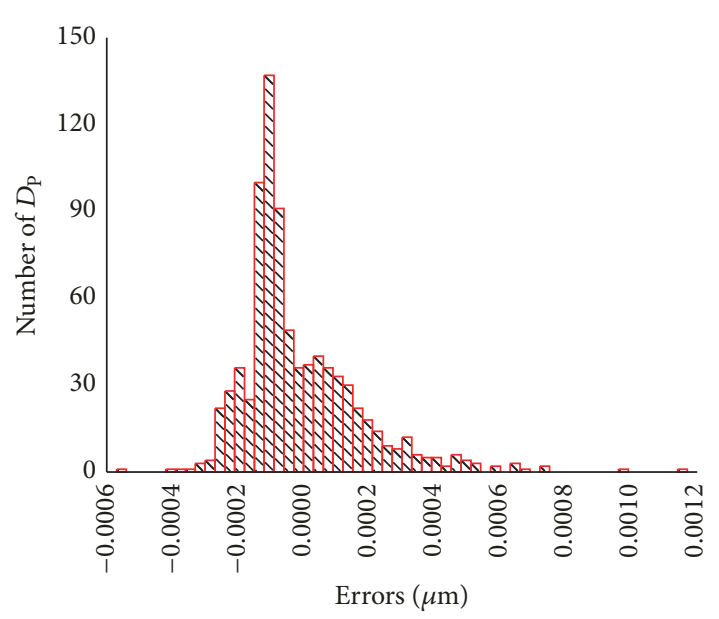

(b) Errors histogram for $D_{\mathrm{P}}$

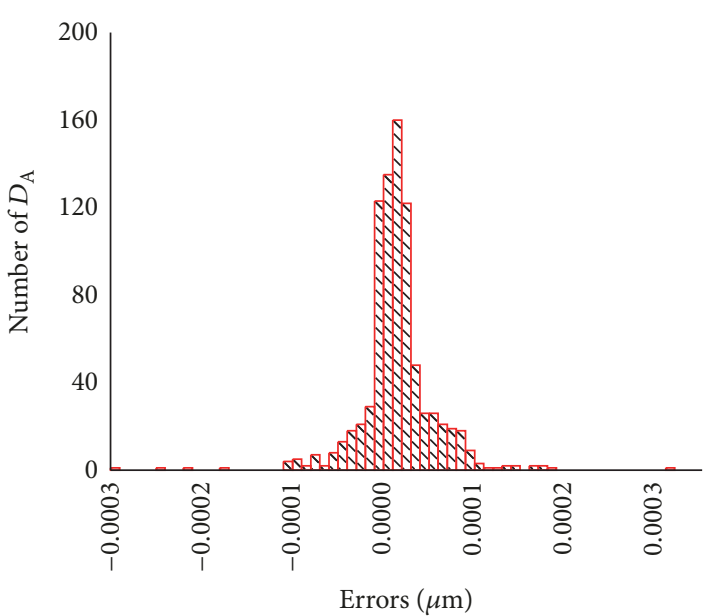

(d) Errors histogram for $D_{\mathrm{A}}$

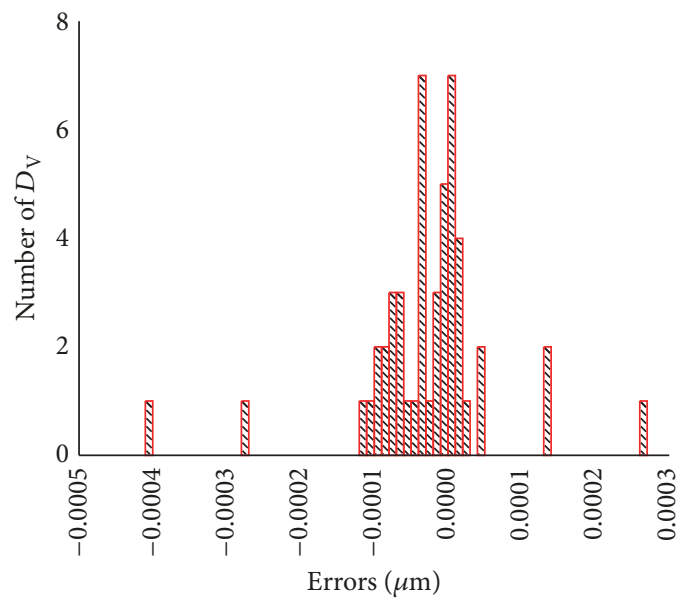

(f) Errors histogram for $D_{\mathrm{V}}$

FIGURE 10: Regression curves and histograms of the trained calculated sizes based on the virtual cylinder with a diameters $D_{\mathrm{vt}}$ of $36 \mathrm{~mm}$ and the "tainbr" function. 


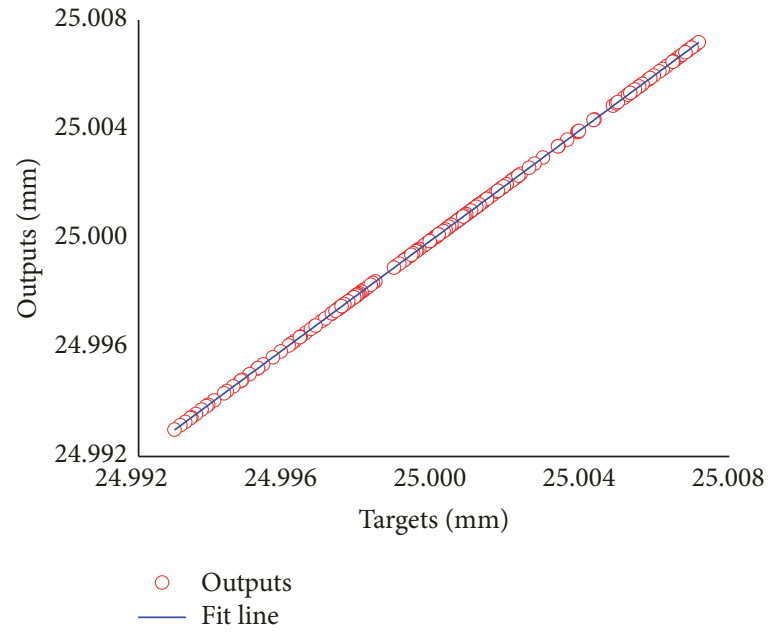

(a) Regression curve for $D_{\mathrm{P}}$

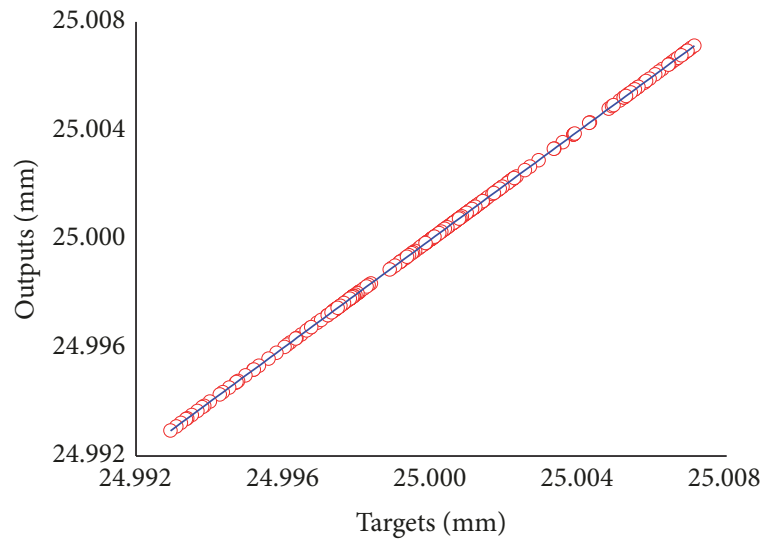

$\bigcirc$ Outputs

— Fit line

(c) Regression curve for $D_{\mathrm{A}}$

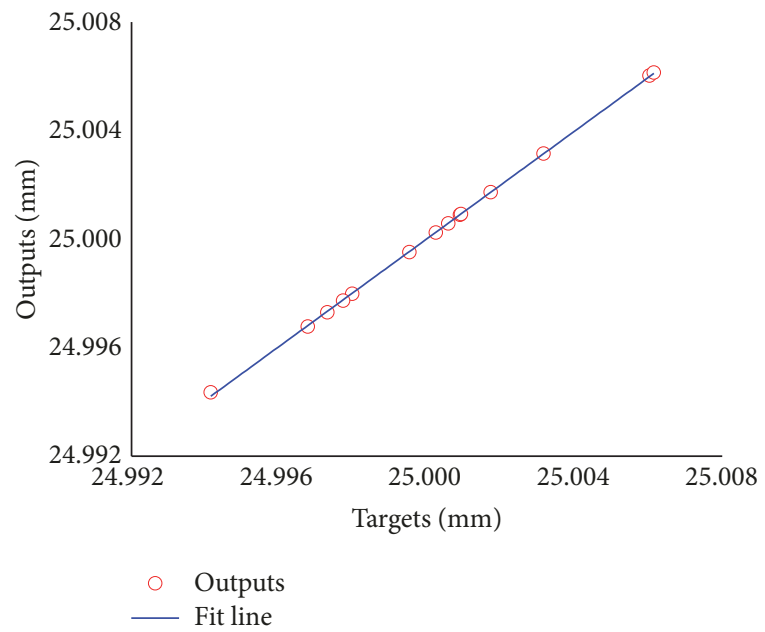

(e) Regression curve for $D_{\mathrm{V}}$

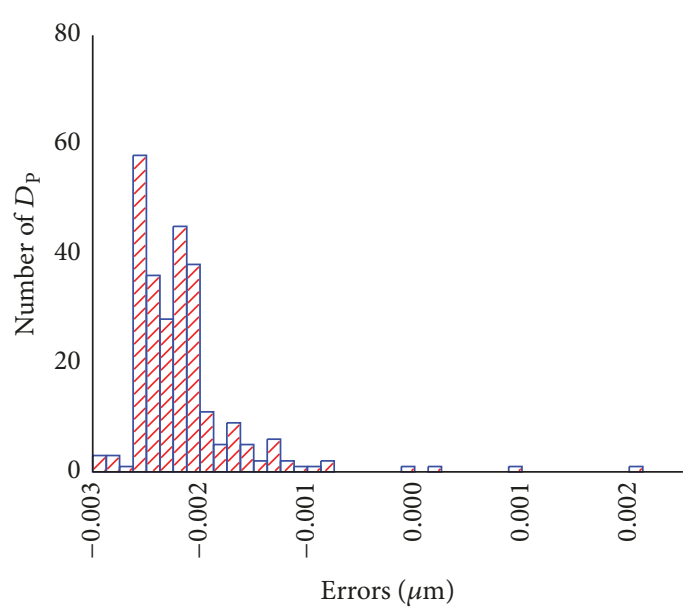

(b) Errors histogram for $D_{\mathrm{P}}$

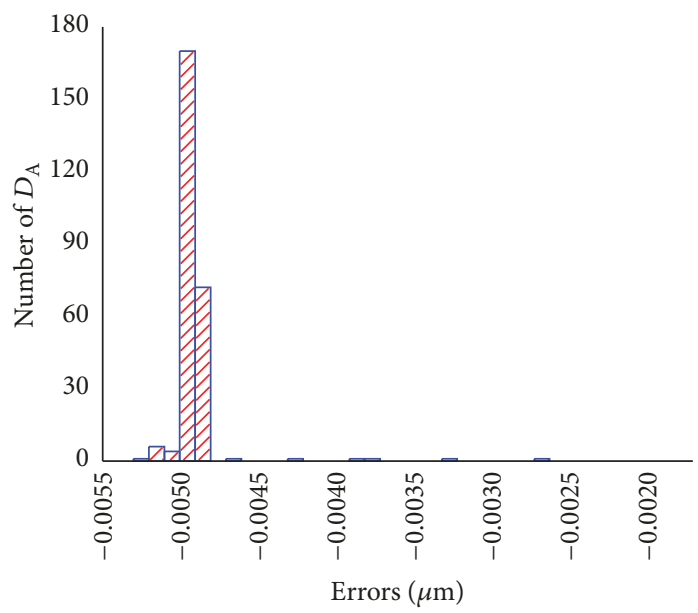

(d) Errors histogram for $D_{\mathrm{A}}$

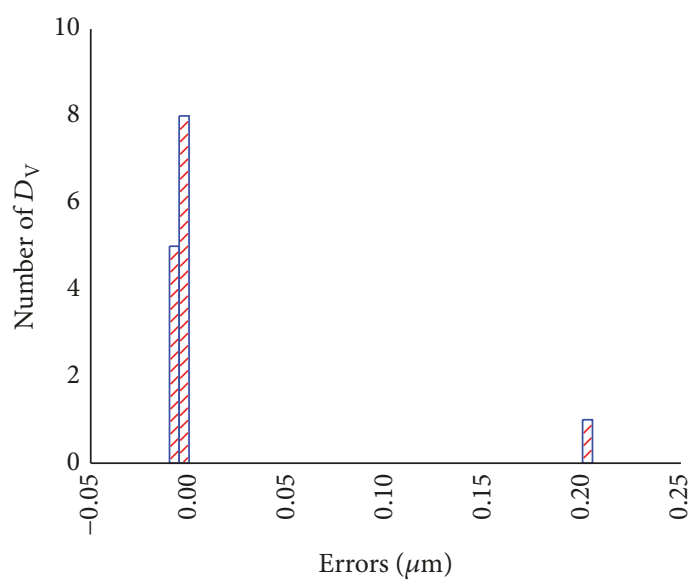

(f) Errors histogram for $D_{\mathrm{V}}$

FIGURE 11: Regression curves and histograms of the trained calculated sizes based on the virtual cylinder with a diameters $D_{\mathrm{vt}}$ of $22.5 \mathrm{~mm}$ and the "tainbr" function. 
where the outputs are the calculated sizes obtained based on the same trained models above and the pseudo calculated sizes of the virtual cylinder with the diameter $D_{\mathrm{vt}}$ of $22.5 \mathrm{~mm}$, and the targets are their corresponding actual calculated sizes. Figures 11(b), 11(d), and 11(f) are the distribution histograms of the errors between the outputs and their corresponding targets.

From Figure 10, the regressions between the targets and the outputs have good linearity, and their errors are very small for 835 roundness profiles of 49 cylinders, which have very high evaluation accuracy. Comparing the regressions and errors in Figures 10 and 11, owing to the fact that the outputs in Figure 10 were obtained by using the neural network regression models based on their roundness profiles and the outputs in Figure 11 were obtained by using the neural network regression models in Figure 10, the regression linearity in Figure 10 is higher than those of their corresponding regressions in Figure 11, and the absolute values of the errors in Figure 10 are less than those of the corresponding errors in Figure 11. Even so, except for one error's absolute value of the volume diameter in Figure 11 which is greater than $0.1 \mu \mathrm{m}$, the absolute values of all the other errors in Figure 11 are far less than $0.1 \mu \mathrm{m}$. Therefore, the evaluation of the calculated sizes based on the trained neural network regression models and the pseudo calculated sizes is feasible and has higher evaluation accuracy.

\section{Conclusions}

Based on the cylindrical coordinate measuring principle, the evaluation models of calculated sizes, such as circumference diameter, area diameter, and volume diameter, were established, by which the pseudo calculated sizes of the virtual cylinders and the checked virtual cylinders and their corresponding actual sizes of the actual cylinders were evaluated. The regression models between the pseudo calculated sizes of the virtual cylinder and their corresponding actual calculated sizes were obtained through a series of neural network training, which were checked by using the calculated sizes of the checked virtual cylinders and their actual corresponding cylinders. The trained and checked experiments for the trained models showed that the neural network regression models trained by using "trainbr" function were better than those trained by using "trainlm" and "trainscg" functions, and the errors between the calculated sizes obtained based on the models trained by using "trainbr" function and the actual calculated sizes were less than $0.1 \mu \mathrm{m}$, which indicated the evaluation method of the calculated sizes based on the neural network regression models is feasible and has higher measuring accuracy.

\section{Data Availability}

The data used to support the findings of this study are available from the corresponding author upon request.

\section{Conflicts of Interest}

The authors declare that they have no conflicts of interest.

\section{Acknowledgments}

This paper was supported by the projects of the National Natural Science Foundation of China (Contracts nos. 51475485 and 51405523).

\section{References}

[1] International Organization for Standardization, ISO 144051:2016 Geometrical Product Specifications (GPS) - Dimensional tolerancing - Part 1: Linear sizes, Geneva, 2016.

[2] G. Goch and K. Lübke, "Tschebyscheff approximation for the calculation of maximum inscribed/minimum circumscribed geometry elements and form deviations," CIRP Annals - Manufacturing Technology, vol. 57, no. 1, pp. 517-520, 2008.

[3] D. A. Masterenko, P. N. Emel'yanov, M. G. Koval'skii, A. Y. Baikovskii, and S. Y. Alabin, "Development of a device for measurement of the deviations of the form and roughness of the surfaces of ultra-precise parts," Measurement Techniques, vol. 57, no. 3, pp. 260-265, 2014.

[4] S. H. R. Ali, H. H. Mohamed, and M. K. Bedewy, "Identifying cylinder liner wear using precise coordinate measurements," International Journal of Precision Engineering and Manufacturing, vol. 10, no. 5, pp. 19-25, 2009.

[5] Z. Zhao, B. Li, G. Zhang et al., "Study on the evaluation of cylinder's global sizes," Precision Engineering, vol. 49, pp. 189199, 2017.

[6] S. Y. Chou and C. W. Sun, "Assessing cylindricity for oblique cylindrical features," The International Journal of Machine Tools and Manufacture, vol. 40, no. 3, pp. 327-341, 2000.

[7] International Organization for Standardization, ISO/TS 121802:2007 Geometrical Product Specifications (GPS) -Cylindricity - Part 2: Specification operators, Geneva, 2007.

[8] M. C. Yoon and D. H. Chin, "Fractal roundness modelling of a measured profile of a cylindrical object," The International Journal of Advanced Manufacturing Technology, vol. 35, no. 1112, pp. 1156-1165, 2008.

[9] A. Rossi, M. Antonetti, M. Barloscio, and M. Lanzetta, "Fast genetic algorithm for roundness evaluation by the minimum zone tolerance (MZT) method," Measurement, vol. 44, no. 7, pp. 1243-1252, 2011.

[10] P. Mohan, P. Haghighi, J. J. Shah, and J. K. Davidson, "Development of a library of feature fitting algorithms for CMMs," International Journal of Precision Engineering and Manufacturing, vol. 16, no. 10, pp. 2101-2113, 2015.

[11] D. S. Srinivasu and N. Venkaiah, "Minimum zone evaluation of roundness using hybrid global search approach," The International Journal of Advanced Manufacturing Technology, vol. 92, no. 5-8, pp. 2743-2754, 2017.

[12] J. Buajarern, T. Somthong, S. H. R. Ali, and A. Tonmeuanwai, "Effect of step number on roundness determination using multi-step method," International Journal of Precision Engineering and Manufacturing, vol. 14, no. 11, pp. 2047-2050, 2013.

[13] K. Lee, S. Cho, and S. Asfour, "Web-based algorithm for cylindricity evaluation using support vector machine learning," Computers \& Industrial Engineering, vol. 60, no. 2, pp. 228-235, 2011.

[14] L. V. Kamble, D. R. Pangavhane, and T. P. Singh, "Neural network optimization by comparing the performances of the training functions -Prediction of heat transfer from horizontal tube immersed in gas-solid fluidized bed," International Journal of Heat and Mass Transfer, vol. 83, pp. 337-344, 2015. 
[15] R. Shaharin, U. K. Prodhan, and M. Rahman, "Performance study of TDNN training algorithm for speech recognition," International Journal of Advanced Research in Computer Science \& Technology, vol. 2, no. 4, pp. 90-95, 2014. 


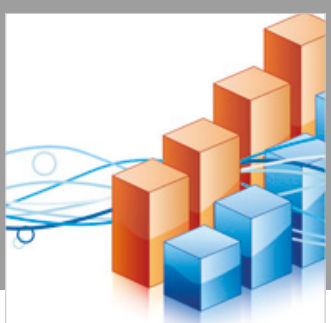

Advances in

Operations Research

\section{-n-m}
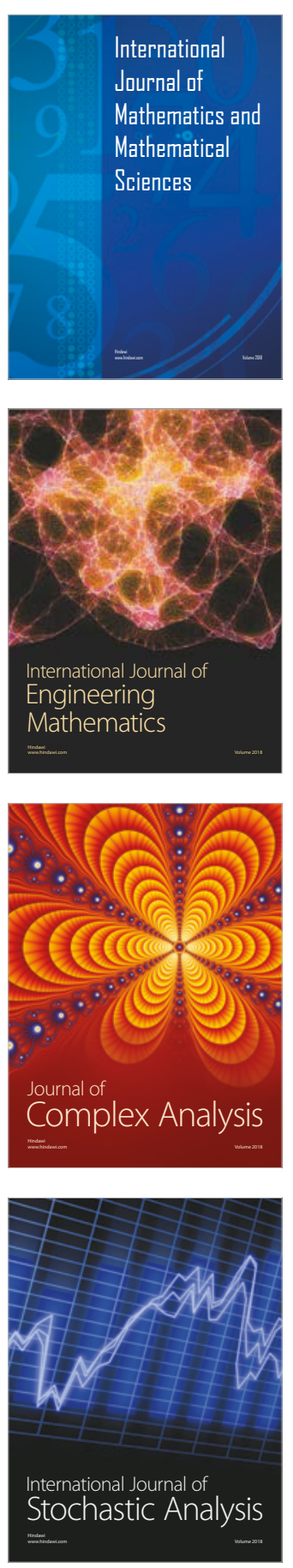
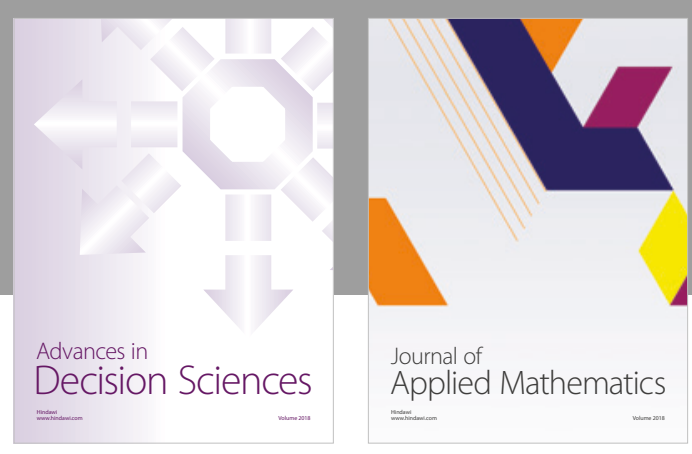

Journal of

Applied Mathematics
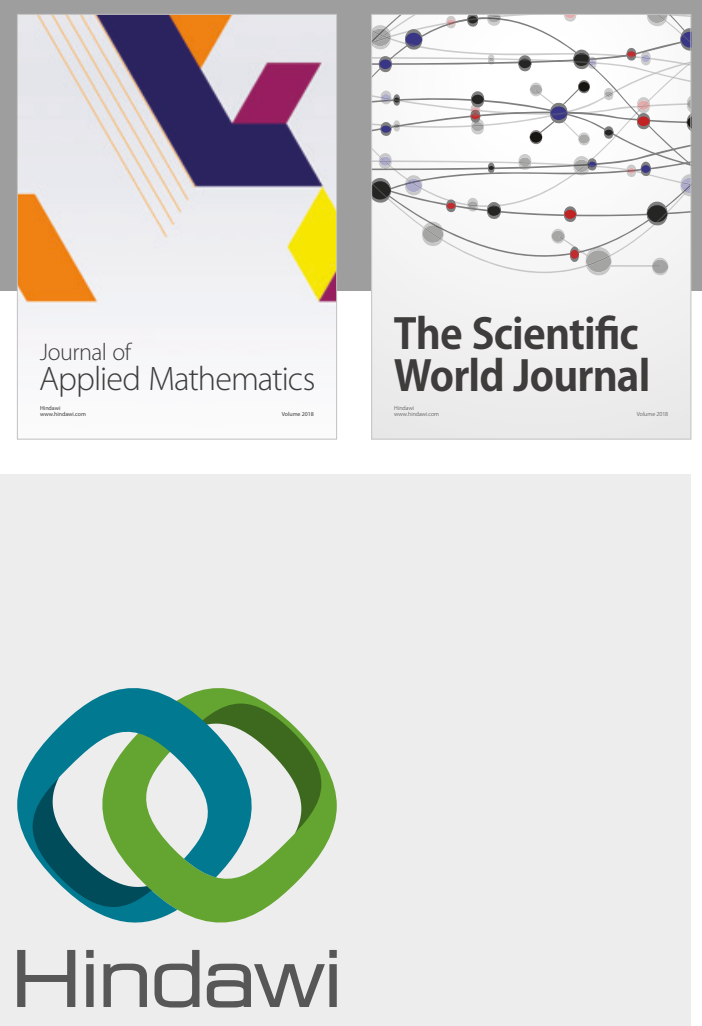

Submit your manuscripts at

www.hindawi.com

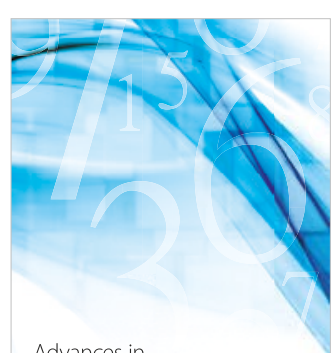

Advances in
Numerical Analysis
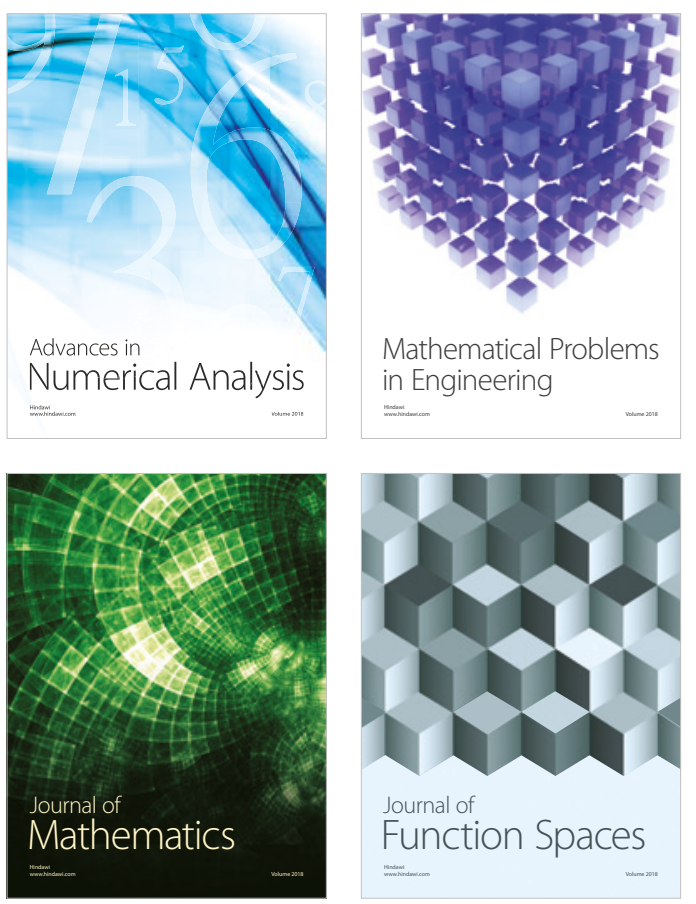

Mathematical Problems in Engineering

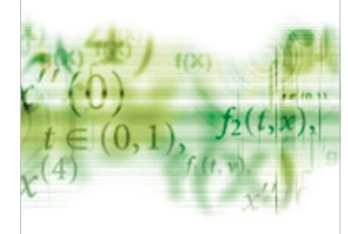

International Journal of

Differential Equations

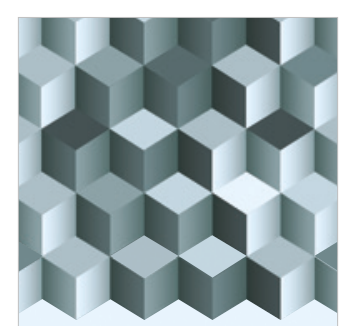

Journal of

Function Spaces

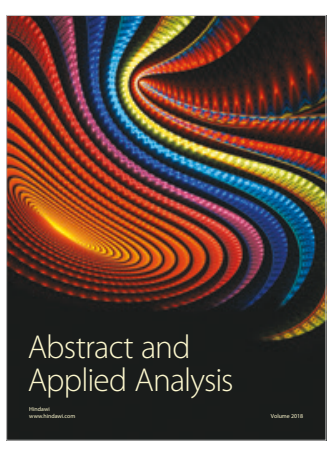

The Scientific

World Journal

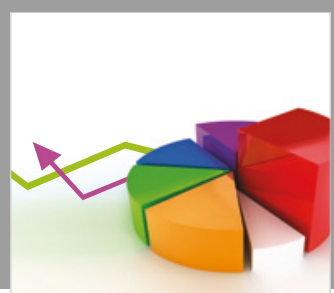

Journal of

Probability and Statistics
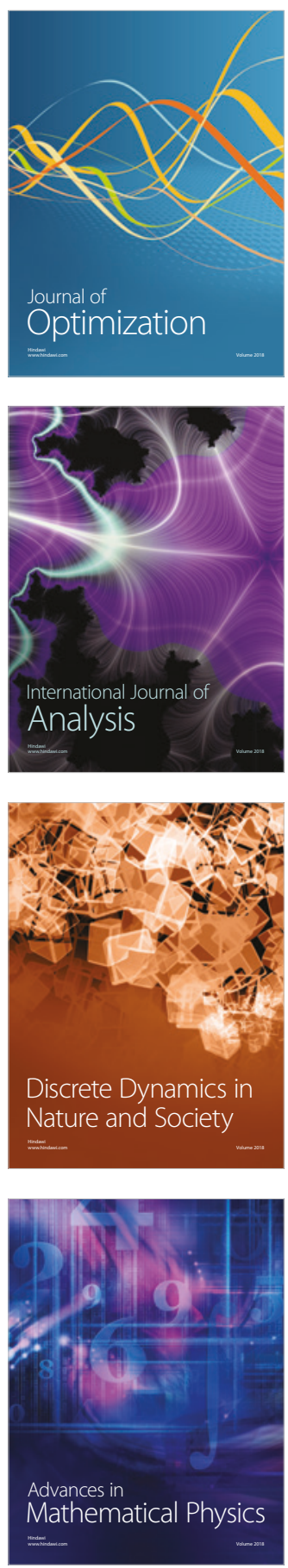